\title{
On the dissolution state of cellulose in cold alkali solutions
}

\author{
Joel Hagman • Luigi Gentile • Christian Moestrup Jessen • \\ Manja Behrens • Karl-Erik Bergqvist • Ulf Olsson
}

Received: 11 January 2017/ Accepted: 14 March 2017/Published online: 30 March 2017

(C) The Author(s) 2017. This article is an open access publication

\begin{abstract}
We have characterized the dissolved state of microcrystalline cellulose (MCC) in cold alkali [2.0 $\mathrm{M} \mathrm{NaOH}(\mathrm{aq})]$ solutions using a combination of small angle X-ray (SAXS) and static light scattering (SLS), ${ }^{1} \mathrm{H}$ NMR, NMR self-diffusion, and rheology experiments. NMR and SAXS data demonstrate that the cellulose is fully molecularly dissolved. SLS, however, shows the presence of large concentration fluctuations in the solution, consistent with significant attractive cellulose-cellulose interactions. The scattering data are consistent with fractal cellulose aggregates of micrometre size having a mass fractal dimension $D \sim 1.5$. At $25^{\circ} \mathrm{C}$ the solution structure remains unchanged on the time scale of weeks. However, upon heating the solutions above $35^{\circ} \mathrm{C}$ additional aggregation occurs on the time scale of minutes. Decreasing or increasing the $\mathrm{NaOH}$ concentration away from the "optimum" $2 \mathrm{M}$ also leads to additional aggregation. This is seen as an increase of the SAXS intensity at lower $q$ values. Addition of urea (1.8 and $3.6 \mathrm{M}$, respectively) does not significantly influence the solution structure. With these examples,
\end{abstract}

J. Hagman $(\bowtie) \cdot$ L. Gentile $\cdot$ C. M. Jessen ·

M. Behrens · U. Olsson

Division of Physical Chemistry, Lund University,

Box 124, 22100 Lund, Sweden

e-mail: joel.hagman@gmail.com

\section{K.-E. Bergqvist}

Centre for Analysis and Synthesis, Lund University,

Box 124, 22100 Lund, Sweden we will discuss how scattering methods can be used to assess the quality of solvents for cellulose.

Keywords Microcrystalline cellulose (MCC) · Cold alkali $(\mathrm{NaOH}) \cdot$ Small angle X-ray scattering (SAXS) . Static light scattering (SLS) · NMR · Rheology · Urea · Co-solvent $\cdot$ Temperature dependence $\cdot$ Aggregation

\section{Introduction}

Cellulose is the world's most abundant polymer and an important natural polymer source for various applications and materials. For applications such as textile fibers, or for doing chemical modifications of cellulose, it is necessary first to dissolve the polymer in a solvent. Finding suitable and environmentally friendly solvents has turned out to be difficult. Cellulose is a crystalline polymer, and due to a stable crystalline state, cellulose is insoluble in common polar solvents, as well as in non-polar and intermediate solvents. In spite of intense work during the last century, only a few types of solvents have been proposed for dissolving cellulose (Liebert 2010; Budtova and Navard 2016). As early as the 1900 s, concentrated $\mathrm{NaOH}(\mathrm{aq})$ was used to dissolve/disperse cellulose in the viscose process. However, $\mathrm{NaOH}(\mathrm{aq})$ can only dissolve cellulose within a narrow $\mathrm{pH}$ range (approximately 1.5-2.5 M NaOH) and only at lower temperatures (typically below $0{ }^{\circ} \mathrm{C}$ ) (Budtova and Navard 2016; 
Alves et al. 2016). The reasons for such limited conditions are still not understood. Additives, like urea, have been reported to facilitate cellulose dissolution in $\mathrm{NaOH}(\mathrm{aq})$ (Liebert 2010; Medronho and Lindman 2014a, b).

The cellulose solution stability is strongly temperature dependent. Roy et al. (2003), in an extensive rheological study, performed time resolved measurements of the linear viscoelastic properties of cellulose solutions in $2.2 \mathrm{M} \mathrm{NaOH}(\mathrm{aq})$ at different temperatures, $15-35^{\circ} \mathrm{C}$. The studied solutions $(5 \mathrm{wt} \%$ cellulose) were initially viscous with the storage modulus, $G^{\prime}$, being smaller than the loss modulus, $G^{\prime \prime}$. At all temperatures, the solutions, after some time, turned into gels with $G^{\prime}>G^{\prime \prime}$, however, with different rates. Defining a gelation time, $t_{\mathrm{gel}}$, as the time when $G^{\prime}=G^{\prime \prime}$, Roy et al. found that $t_{\text {gel }}$ decreased exponentially with increasing temperature. The reason for this gelation is yet not fully understood (Budtova and Navard 2016).

To characterize the dissolution state of macromolecules in solution scattering studies, i.e., small angle scattering of X-rays (SAXS) or neutrons (SANS), combined with light scattering, are particularly useful as they can report on the solution structure on the colloidal length scale, 1-1000 nm. Recently we have characterized the dissolution state of microcrystalline cellulose, MCC, in the related solvent, $40 \mathrm{wt} \%$ tetrabutyl ammonium hydroxide in water, TBAH(aq) (Gubitosi et al. 2016; Gentile and Olsson 2016; Behrens et al. 2016). It was found that the cellulose was molecularly dissolved, with effectively repulsive cellulose-cellulose interactions at concentrations lower than ca. $4 \mathrm{wt} \%$. At higher concentration, however, aggregates were formed indicating effectively repulsive interactions. It was proposed that ca. $4 \mathrm{wt} \%$ marked the solubility of Cellulose II, the crystal polymorph that often forms when regenerating or mercerizing cellulose. Natural cellulose, Cellulose I, is expected to be less stable than Cellulose II and hence have a higher solubility (Behrens et al. 2016).

In the present paper we have similarly characterized the dissolution state of cellulose in aqueous $\mathrm{NaO}$ $\mathrm{H}(\mathrm{aq})$, using light scattering and SAXS. Mainly in 2.0 $\mathrm{M} \mathrm{NaOH}(\mathrm{aq})$, but also varying the $\mathrm{NaOH}$ concentration. We also studied the effect of urea on the dissolution state. To further characterize the cellulose solutions we also present data on shear viscosity, ${ }^{1} \mathrm{H}$ NMR and cellulose self-diffusion experiments, using pulsed gradient NMR.

\section{Materials and methods}

\section{Materials}

Microcrystalline cellulose, Avicel PH101, Lot: 61113C, Box 00083, was purchased from SigmaAldrich. Sodium hydroxide, $\geq 97 \%$ pure, anhydrous pellets, was purchased from Merck, Darmstadt, Germany. Sodium deuteroxide, $40 \mathrm{~g} / \mathrm{cm}^{3}$ in $\mathrm{D}_{2} \mathrm{O}$, 99.5 at.\% D, was purchased from Sigma-Aldrich. $\mathrm{D}_{2} \mathrm{O}, 99.8$ at.\%, Lot: 10468, was purchased from ARMAR Chemicals, Döttingen, Switzerland. The water used was purified in-house using a MILLIPORE Milli-Q Gradient A 10, Millipore, Molsheim, France.

\section{Sample preparation}

MCC was dissolved in cold alkali solutions using the following procedure: A solution of $2.0 \mathrm{M} \mathrm{NaOH}$ (prepared as stock solution by dissolving solid $\mathrm{NaOH}$ pellets in Milli-Q water) was pre-cooled in an ice bath and the MCC was then slowly added to the solution under vigorous stirring using a magnet stirrer $(600 \mathrm{rpm})$. The dispersion was left to stir in the ice bath until the appearance was that of a semitransparent, homogeneous dispersion $(\geq 5 \mathrm{~min})$ and then subsequently put in a freezer $\left(-20^{\circ} \mathrm{C}\right)$ for $20 \mathrm{~min}$. Once the sample had undergone the freezing step, it was brought back to room temperature by first letting it stir in an ice bath for $10 \mathrm{~min}$ and then left to stir without any temperature control for an additional $20 \mathrm{~min}$. After the final stirring, the samples were ready and kept at room temperature. For SAXS measurements, the samples were used as prepared or diluted to the desired concentration. For light scattering, the stock solution of $\mathrm{NaOH}$ was filtered through an Acrodisic syringe filter with a $0.2 \mu \mathrm{m}$ Super membrane before use and the ready samples were centrifuged at $13 \mathrm{k}$ G for $6 \mathrm{~h}$ prior to dilution. Samples for NMR measurements were made using the same protocol in $\mathrm{NaOD} / \mathrm{D}_{2} \mathrm{O}$, at the same molar $(2.0 \mathrm{M})$ concentration of NaOD. In Table 1 there is an overview of the experiments performed. 
Table 1 Overview of the experiment parameters

\begin{tabular}{llll}
\hline Experiment & Conc. range $\left(\mathrm{g} / \mathrm{cm}^{3}\right)$ & Temp. range $\left({ }^{\circ} \mathrm{C}\right)$ & Sample prep \\
\hline SLS & $0.0025-0.01$ & 25 & Diluted from $0.010 \mathrm{~g} / \mathrm{cm}^{3} \mathrm{stem}$ \\
SAXS conc. series & $0.0025-0.01$ & 25 & Diluted from $0.010 \mathrm{~g} / \mathrm{cm}^{3} \mathrm{stem}$ \\
SAXS temp. series & $0.0025-0.01$ & $25-45$ & Diluted from $0.020 \mathrm{~g} / \mathrm{cm}^{3} \mathrm{stem}$ \\
SAXS NaOH conc & $0.0025-0.01$ & 25 & Diluted from $0.020 \mathrm{~g} / \mathrm{cm}^{3} \mathrm{stem}$ \\
Rheology conc series & $0.0025-0.02$ & 25 & Diluted from $0.020 \mathrm{~g} / \mathrm{cm}^{3} \mathrm{stem}$ \\
Rheology temp. series & $0.0025-0.02$ & $25-45$ & Diluted from $0.020 \mathrm{~g} / \mathrm{cm}^{3} \mathrm{stem}$ \\
Rheology NAOH conc & $0.0025-0.02$ & 25 & Diluted from $0.020 \mathrm{~g} / \mathrm{cm}^{3} \mathrm{stem}$ \\
NMR & $0.005-0.02$ & 25 & Individually prepared samples \\
${ }^{1} \mathrm{H}-\mathrm{NMR}$ & $0.005-0.02$ & 25 & Individually prepared samples \\
\hline
\end{tabular}

Static light scattering (SLS)

For SLS measurements, we used an ALV/DLS/SLS5022F, CGF-8F-based compact goniometer system from ALV-GmbH, Langen, Germany with a $22 \mathrm{~mW}$ $\mathrm{He}-\mathrm{Ne}$ laser as light source. The laser operates at $632.8 \mathrm{~nm}$, and the intensity is varied using a softwarecontrolled attenuator. A perfect vertical polarisation is achieved using a Glan laser polarizer prism with a polarization ratio better than $10^{5}$ in front of the temperature controlled cell housing. The scattering cells used were made of borosilicate glass $(10 \mathrm{~mm}$ inner diameter) and were immersed in a thermostated vat filled with a refractive index matched liquid (cisdecahydronaphthalene), and the temperature was controlled using a F32 Julabo heating circulator which kept an accuracy of $\pm 0.1{ }^{\circ} \mathrm{C}$. The unpolarised scattered light is collected using a detection unit that includes a near-monomodal optical fibre and two highquality avalanche photodiodes placed in a pseudocross geometry. The rotary table of the goniometer has a range of scattering angles $(\theta)$ between $20^{\circ}$ and $140^{\circ}$.

\section{Small-angle X-ray scattering (SAXS)}

Small-angle X-ray scattering measurements were performed using the SAXSLab Ganesha 300XL instrument (SAXSLAB ApS, Skovlunde, Denmark), a pinhole collimated system equipped with a Genix 3D X-ray source (Xenocs SA, Sassenage, France). Data were collected with the detector placed at various sample-to-detector positions that yielded to an overall $q$ range of $4.1 \times 10^{-3}-7.3 \times 10^{-1} \AA^{-1}$. Samples were sealed at room temperature in a $1.5 \mathrm{~mm}$ diameter quartz capillary (Hilgenberg $\mathrm{GmbH}$, Malsfeld,
Germany). In all cases the temperature was controlled by an external recirculating water bath with an accuracy of $\pm 0.2^{\circ} \mathrm{C}$. The two-dimensional (2D) scattering pattern was recorded using a $2 \mathrm{D} 300 \mathrm{k}$ Pilatus detector (Dectris Ltd., Baden, Switzerland) and radially averaged using SAXSGui software to obtain $I(q)$. The measured scattering curves were corrected for solvent scattering.

\section{NMR diffusion}

NMR diffusion experiments were performed on a Bruker Avance DMX200 spectrometer operating at 200.13 $\mathrm{MHz}$ on ${ }^{1} \mathrm{H}$ nuclei was equipped with a commercial diffusion probe (DIF-25 $5 \mathrm{~mm}$ ) having a maximum gradient strength of $960 \mathrm{G} \mathrm{cm}^{-1}$. Experiments were performed using the stimulated echo sequence (Price 2009), with field gradient pulses of duration $\delta=2 \mathrm{~ms}$, and separation $\Delta=30 \mathrm{~ms}$, and varying the gradient strength, $\mathrm{G}$, in the range from 107 to $428 \mathrm{G} \mathrm{cm}^{-1}$. The stimulated echo amplitude is given by

$$
E=0.5 \times e^{-t_{23} / T_{1}} \times e^{-2 t_{12} / T_{2}} \times e^{-k D},
$$

where $t_{12}=3.16 \mathrm{~ms}$ and $t_{23}=26.8 \mathrm{~ms}$ are the waiting times between the first and second and second and third radio frequency (RF) pulses, respectively. $T_{1}$ and $T_{2}$ are the longitudinal and transverse relaxation times, respectively; $t_{12}$ is short and, therefore, there is no $T_{2}$ relaxation. $D$ is the self-diffusion coefficient and $k$ is given by

$k=(\gamma G \delta)^{2}(\Delta-\delta / 3)$,

where $\gamma$ is the magnetogyric ratio. Cellulose selfdiffusion was determined from the echo decay of the 
$3.6 \mathrm{ppm}$ peak (Fig. 4a). The temperature was kept constant $\left(25^{\circ} \mathrm{C}\right)$ using conventional Bruker temperature control, i.e., dry air was used as variable temperature control gas. The sample was allowed to equilibrate at each desired temperature for $15 \mathrm{~min}$ before acquisition. The accuracy of the temperature control was $\pm 0.4{ }^{\circ} \mathrm{C}$.

\section{${ }^{1} H N M R$}

The ${ }^{1} \mathrm{H}$ spectra were recorded on a Bruker DRX 500 spectrometer (Bruker, Germany), equipped with a $5 \mathrm{~mm}$, broadband probe optimized for $1 \mathrm{H}$ observation. The residual HDO peak was used as a reference to calibrate the chemical shifts. Spectra were collected using a $90^{\circ}$ pulse (12 $\mu$ s pulse duration), an acquisition time of $200 \mathrm{~ms}$, a relaxation delay of $2 \mathrm{~s}$, and 1000 scans. Spectra were processed using topspin 2.0 (Bruker) with a line broadening of $2 \mathrm{~Hz}$.

\section{Rheology}

Rheological measurements have been carried out using Anton Paar Physica MCR 301 (Anton Paar, Germany) a stress controlled rheometer with direct strain oscillation (DSO) for real-time strain control equipped with a $2^{\circ}$ cone-plate geometry, a diameter of $49.98 \mathrm{~mm}$ and truncation of $208 \mu \mathrm{m}$. The temperature has been controlled by a Peltier temperature control with actively heated Peltier hood (US Patent $6,571,610)$. To prevent errors due to evaporation, a solvent trap containing water has surrounded the measuring geometry. Stationary experiments have been performed to obtain flow curves. The flow curves were obtained increasing the shear rate from 0.1 to 100 $\mathrm{s}^{-1}$ in a logarithmic scale.

\section{Results and discussion}

\section{Cellulose in $2.0 \mathrm{M} \mathrm{NaOH}(\mathrm{aq})$ at $25^{\circ} \mathrm{C}$}

Dilute cellulose solutions in $2.0 \mathrm{M} \mathrm{NaOH}(\mathrm{aq})$ were studied at $25^{\circ} \mathrm{C}$ using small angle X-ray scattering, SAXS, and static light scattering, SLS, and in addition to viscosity and NMR self-diffusion experiments. In Fig. 1, we present results from these different techniques for a cellulose concentration of $0.010 \mathrm{~g} / \mathrm{cm}^{3}$. The scattered intensity, $I(q)$, can be written as
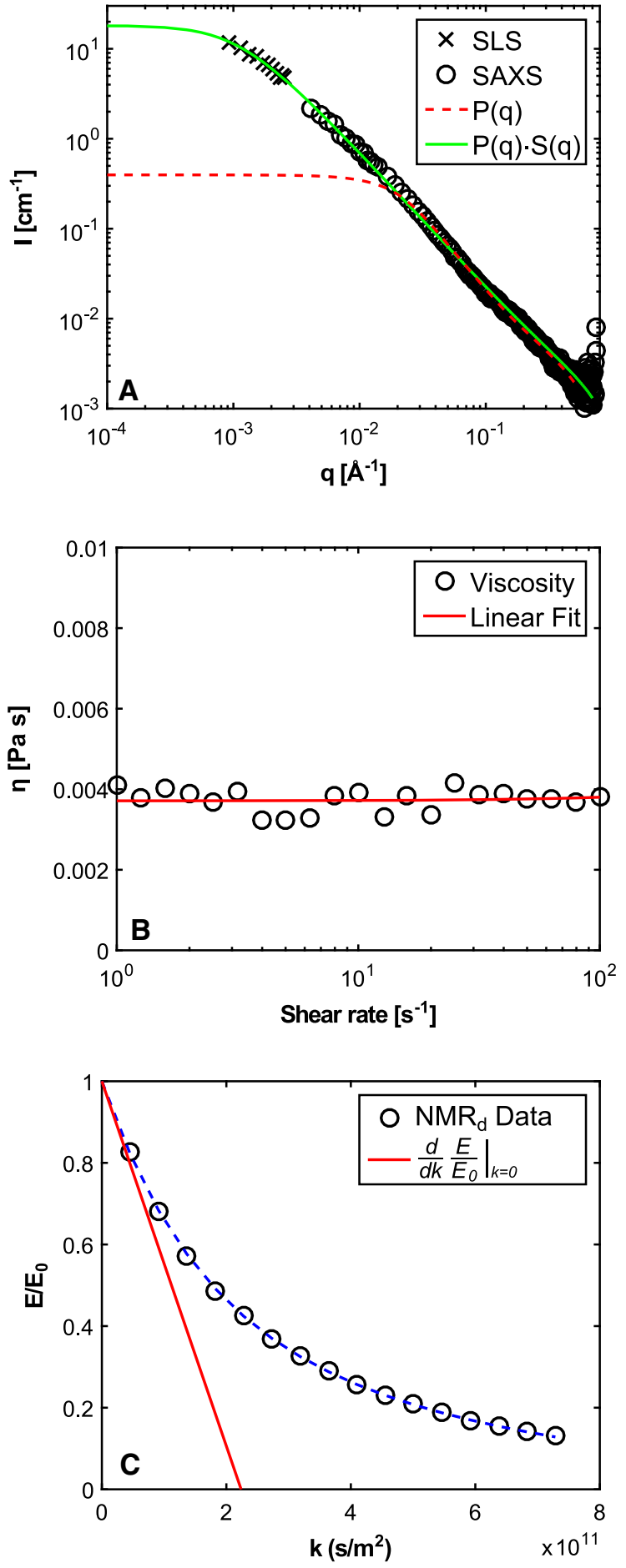

$I(q)=K c M_{\mathrm{w}} P(q) S(q)$.

Here, $q=(4 \pi n / \lambda) \sin (\theta / 2)$ is the magnitude of scattering vector, $n$ is the refractive index of the solution, $\lambda$ is the radiation wave length in vacuum and 
४Fig. 1 Experimental results obtained for $0.010 \mathrm{~g} / \mathrm{cm}^{3}$ cellulose solution in $2.0 \mathrm{M} \mathrm{NaOH}(\mathrm{aq})$ at $25^{\circ} \mathrm{C}$. a Experimental scattering pattern, SAXS (open circle) and SLS (cross), together with a calculated form factor, $P(q)$ (dashed red line), and a model including a structure factor $S(q)$ describing fractal aggregation (solid green line). b Shear viscosity plotted as a function of shear rate. c Echo decay from a NMR diffusion experiment. The dashed blue line represents a bi-exponential decay fitted to the data to guide the eye. The solid red line is a fit of the initial slope of the echo decay from which an average diffusion coefficient is obtained

$\theta$ is the scattering angle. $M_{\mathrm{w}}$ is the weight-averaged molecular weight and $c$ is the concentration of the solute. $P(q)$ is the average form factor, averaged over the polydisperse cellulose population, and $S(q)$ is the effective structure factor. $K$ is an optical constant which for SLS is given by

$$
K_{\mathrm{SLS}}=\frac{4 n_{0}^{2} \pi^{2}}{N_{\mathrm{A}} \lambda^{4}}\left(\frac{\mathrm{d} n}{\mathrm{~d} c}\right)^{2},
$$

where $N_{\mathrm{A}}$ is the Avogadro constant, $N_{0}$ is the solvent refractive index, and the refractive index increment, $\mathrm{d} n / \mathrm{d} c$, is the scattering contrast. For SAXS the optical constant is given by

$$
K_{\mathrm{SAXS}}=\frac{\Delta \varrho^{2}}{\rho^{2} N_{\mathrm{A}}},
$$

where $\rho=1.5 \mathrm{~g} / \mathrm{cm}^{3}$ is the mass density of cellulose, and $\Delta \varrho=3.8 \times 10^{10} \mathrm{~cm}^{-2}$ is the scattering length difference between cellulose and solvent.

A combination of absolute scaled SAXS and SLS data of a $0.010 \mathrm{~g} / \mathrm{cm}^{3}$ sample is presented in Fig. 1a. Here, the SLS data have been converted to X-ray contrast by multiplying the absolute scaled data with $K_{\text {SAXS }} / K_{\text {SLS. }}$ As a comparison, we show as a dashed curve the pure form factor scattering, i.e., assuming $S(q)=1$ for semi flexible chains with excluded volume interactions. This parametrized form factor was derived by Pedersen and Schurtenberger (1996) from computer simulations (Chen et al. 2006). In the present calculation we have used the experimentally determined $M_{\mathrm{w}}=29 \mathrm{~kg} / \mathrm{mol}$ corresponding to an average contour length of $\langle L\rangle \approx 82 \mathrm{~nm}$, assuming a length of $0.515 \mathrm{~nm}$ (Schulz et al. 2000) for every glucose unit, a cross sectional radius of $0.25 \mathrm{~nm}$ and a Kuhn length $l_{\mathrm{K}}=2.3 \mathrm{~nm}$ (corresponding to approximately 4 glucose units). Here, the value of $l_{\mathrm{K}}$ was adjusted to fit the experimental data at higher $q$.
As clearly seen in Fig. 1a, the form factor alone is unable to describe the observed scattering over the whole $q$ range. Experimentally we observe a significantly higher scattering at lower $q$ values, signalling the presence of effectively attractive cellulose-cellulose interactions resulting in very large concentration fluctuations or possibly rather the formation of cellulose "clusters". To model also the low q data we have adopted the fractal aggregation $S(q)$ of Teixeira (1988),

$$
S(q)=1+\frac{D \Gamma(D-1) \sin \left[(D-1) \tan ^{-1}(q \xi)\right]}{(q r)^{D}\left[1+(q \xi)^{-2}\right]^{(D-1) / 2}} .
$$

Here, $\Gamma(x)$ is the gamma function, $D$ is the fractal dimension, $\xi$ is the fractal correlation length, i.e., the effective cluster size, and $r$ is an effective size of the aggregating objects. The solid line in Fig. 1a is a model calculation where we have also included $S(q)$ according to Eq. (6), with $D=1.5, \xi=103 \mathrm{~nm}$ and we use a radius equal to the cross section of the cellulose chain $(0.25 \mathrm{~nm})$. As can be seen, this allows for a good description of the data over the whole $q$ range.

The sample has Newtonian flow behaviour. In Fig. 1b we present the measured shear viscosity, $\eta(\dot{\gamma})$, plotted as a function of the shear rate, $\dot{\gamma}$. As can be seen, $\eta$ is independent of $\dot{\gamma}$, and we measure a zero-shear viscosity $\eta_{0}=3.7 \mathrm{mPas}$, corresponding to $\eta_{\text {rel }}=$ $\eta_{0} / \eta_{s}=2.54$, where $\eta_{\mathrm{s}}=1.455 \mathrm{mPas}$ is the solvent $(2.0 \mathrm{M} \mathrm{NaOH}(\mathrm{aq}))$ viscosity. This value is fairly close to the value of roughly 2.7 , reported by Roy et al. (2003) for $1 \mathrm{wt} \% \mathrm{MCC}$ in a $9 \mathrm{wt} \% \mathrm{NaOH}$ solution.

The self-diffusion of cellulose was measured by pulsed gradient NMR. In Fig. 1c we present the relative echo amplitude, $E / E_{0}$, as a function of the parameter $k=(\gamma \delta G)^{2}(\Delta-\delta / 3)$ [Eq. (2)]. $\Delta=30 \mathrm{~ms}$ is the separation of the two gradient pulses, and corresponds to the observation time scale of the experiment. For a single component, the echo decay is exponential, $E / E_{0}=\mathrm{e}^{-k D}$, with $D$ being the diffusion coefficient. In the polydisperse case, the echo decay becomes a weighted sum of exponentials, summing up all the molecular weights in the solution. In the stimulated echo sequence (Price 2009), with $t_{12}<<T_{2}$ and $t_{23}<<T_{1}$, spin relaxation does not influence the relative weights of the different molecular weights and the relative echo amplitude is given by 


$$
\frac{E}{E_{0}}=\frac{\sum_{i} n_{i} M_{i} e^{-k D_{i}}}{\sum_{i} n_{i} M_{i}} .
$$

Here, $n_{i}$ is the number of molecules of molar mass $M_{i}$ in the solution, and we note that the NMR signal from each molecule is proportional to its size, i.e., $M_{i}$. In dilute solutions, $D_{i} \sim 1 / R_{\mathrm{H}_{i}}$ and $1 / R_{\mathrm{H}_{i}} \sim M_{i}^{1 / 2}$. For a broad size distribution, say in the range 1000-100,000 $\mathrm{g} / \mathrm{mol}$ as for the case of the MCC used, it is expected to be approximately an order of magnitude difference between the lowest and highest diffusion coefficient. A reasonable description of the size distribution requires sampling of the echo decay over at least two orders of magnitude in order to fit the echo-decay using a sum of exponentials; i.e., bring $E / E_{0}$ down to 0.01 or below. This is not the case in Fig. 1c. A simpler alternative is to only make single parameter fit and deduce an average diffusion coefficient from the initial slope. The first derivative of $E / E_{0}$ can be written as

$$
\left.\frac{\mathrm{d}}{\mathrm{d} k} \frac{E}{E_{0}}\right]_{k=0}=-\langle D\rangle_{\mathrm{w}}
$$

and thus corresponds to the mass weighted averaged self-diffusion coefficient, from which we can calculate the mass weighted average hydrodynamic radius. The $\langle D\rangle_{\mathrm{w}}$ is thus obtained without any assumption of the size distribution and is hence a robust measurement.

The data in Fig. 1c is fitted with a bi-exponential curve, which reflects on the polydispersity of the sample, and the derivative of the initial slope gives $\langle D\rangle_{\mathrm{w}}=4.5 \times 10^{-12} \mathrm{~m}^{2} / \mathrm{s}$. From the self-diffusion coefficient we can calculate an apparent hydrodynamic radius from the Stokes-Einstein relation

$$
D=\frac{k_{\mathrm{B}} T}{6 \pi \eta R_{\mathrm{H}}}
$$

which corresponds to an average apparent hydrodynamic radius $\left\langle R_{\mathrm{H}}\right\rangle_{\mathrm{w}}=33.6 \mathrm{~nm}$, calculated by using $\eta=1.455 \mathrm{mPas}$, from $\mathrm{NaOH}$ as no data for deuterated environment was found.

This is seems to be in disagreement with the scattering data, which suggests $\mu \mathrm{m}$ sized aggregates. However, the echo decay is only taken down to 0.13 , which means that it is only possible to follow the diffusion of $87 \%$ of all species in the sample. The scattering techniques report the intensity weighted information and the intensity measured scales with the size of the molecules, but not with the amount. It is, thus, fully possible that a vast majority of the molecules are able to move around more or less freely and that the very large clusters seen with SLS and SAXS are simply so slow that they are not seen by NMR. Another explanation is the structures suggested by Schulz et al. (2000). They suggests aggregated fringed micelles, where large clusters form with a lot of free chains sticking out. This type of structure could be thought of as a "fluffy" cluster, which scatters light in SAXS and SLS, but still allows individual chains to diffuse relatively freely and unhindered around the aggregate. A third option would be that the "clusters" seen are, in fact, not clusters at all, but instead concentration fluctuations. That is, the cellulose chains are molecularly dissolved, but diffuse around and randomly creates local density fluctuations. This would cause regions, which would scatter light as if they were large clusters, but since no cellulose chains would be bound, individual chains would still be able to diffuse around freely and unhindered through the regions with higher concentration of cellulose.

\section{Sample stability}

Sample stability may be an issue as the alkali environment may degrade the cellulose chains and since the fully prepared samples have been kept at room temperature $\left(22 \pm 1{ }^{\circ} \mathrm{C}\right)$ during analysis, a temperature in which the MCC is not fully stable in $\mathrm{NaOH}$ solutions (Cai and Zhang 2006; Chen et al. 2007), it is also important to verify that there are no changes occurring on the timescale of an experiments. Roy et al. (2003) performed gelation experiments on cellulose in $9 \mathrm{wt} \% \mathrm{NaOH}$ solution, but only showed kinetics for a $5 \mathrm{wt} \%$ cellulose concentration which gelled after roughly $50 \mathrm{~h}$ in room temperature. The difference in cellulose concentration makes their timescale difficult to relate to the one for a solution of $0.010 \mathrm{~g} / \mathrm{cm}^{3}$ ( $\sim 1 \mathrm{wt} \%$ ); there should be no doubt that our samples would gel eventually in room temperature, but they are stable far longer than $50 \mathrm{~h}$.

Figure 2 demonstrates the change in scattering intensity with time for a fresh sample, the same sample after 3 weeks, as well as after 2 months, and, as can be seen, no significant changes are recorded. The lack of changes in intensity within the analysed q-range on a timescale of up to 2 months means that any potential degradation of the MCC chains can be neglected 


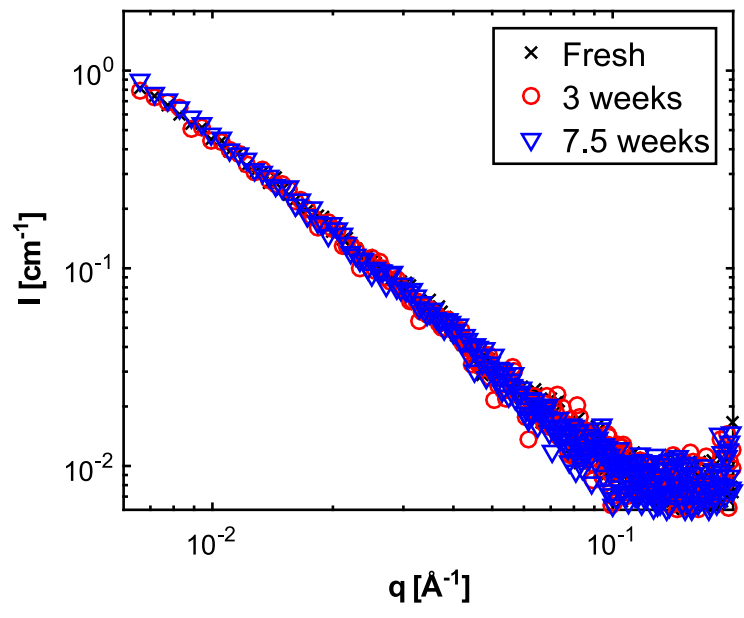

Fig. 2 SAXS pattern from a $0.010 \mathrm{~g} / \mathrm{cm}^{3}$ sample recorded just after preparation (cross), after 3 weeks (open circle), and after 7.5 weeks (upside down triangle)

during the timescale of an average analysis (hours). Several studies on the degradation in $\mathrm{NaOH}$ solution have been performed and the data in the review article by Loon and Glaus (1997) suggests that no more than $10 \%$ of the cellulose chains should become degraded over the course of 60 days.

\section{MCC concentration dependency}

The concentration dependence of the samples is rather interesting. If the data in Fig. 1 can be explained by concentration fluctuations of the cellulose, then it is reasonable to assume that the system should change upon dilution. The scattering data in Fig. $3 \mathrm{a}$ are normalized by the concentration and the curves overlap well, both for the SAXS and for SLS regions. If long lived aggregations are formed, then it is to be expected that they remain the same size and quantity upon dilution, and thus the intensity normalized scattering should remain constant. In the case that there are no permanent aggregations forming between the cellulose chains, there should be a decrease in concentration normalized scattering as each polymer should, on average, come further away from its neighbours a result of dilution. That is, the regions with higher concentration should disappear with dilution as the average distance between chains becomes larger and an intensity normalized scattering should, therefore, decrease. As Fig. 3a shows, this is not the case and instead the concentration normalized scattering is unchanged even in the very low q region of light scattering.

Figure $3 \mathrm{~b}$ present the relative zero shear viscosity as a function of concentration and the data follows a quadratic relation for the range of $0.0025-0.020 \mathrm{~g} /$ $\mathrm{cm}^{3}$. The have been fitted with a truncated version of Huggins equation (Huggins 1942), such as

$$
\frac{\eta}{\eta_{0}}=1+[\eta] c+K_{\mathrm{H}}([\eta] c)^{2}
$$

where $\eta$ is the zero shear viscosity of the solution, $\eta_{0}$ is the zero shear viscosity of the solvent, $[\eta]$ is the intrinsic viscosity, $c$ is the solution concentration, and $K_{\mathrm{H}}$ is the Huggins constant. The fit is mainly intended as a guide for the eye and cannot reliably be used to estimate the parameters due to the low amount of data points. However, the fit gave a value of $[\eta]$ of $21 \mathrm{~cm}^{3} /$ $\mathrm{g}$, which, if it is a true estimate, would yield a critical overlap concentration, $C^{*}$, of $0.048 \mathrm{~g} / \mathrm{cm}^{3}$ based on $C^{*} \sim 1 /[\eta]$. This value fits well with other estimates of $C^{*}$ of the same MCC (Behrens et al. 2016) and Roy et al. (2003) obtains a similar concentration dependence for MCC in a $9 \mathrm{wt} \% \mathrm{NaOH}$ solution. The increase in relative viscosity seen is an indication of attractive interactions between the cellulose chains as more and more chains have the chance to interact with each other with increasing concentration. The concentration dependence of the self-diffusion $\left(\langle D\rangle_{\mathrm{w}}\right.$, $\left.\mathrm{NMR}_{d}\right)$ and the z-averaged collective diffusion coefficient $\left(\left\langle D_{\mathrm{c}}\right\rangle_{z}\right.$, DLS) are presented in Fig. 3c. The difference between the two datasets is that the $\mathrm{NMR}_{d}$ captures the diffusion of the single chains whereas DLS captures the collective movement and the trend for both data sets is fitted with a solid and dashed line respectively. The decrease of the diffusion coefficients with increasing concentration is consistent with increasing aggregation and effective attractive cellulosecellulose interactions. The free, unobstructed $\langle D\rangle_{\mathrm{w}}$ can be obtained by extrapolating to $\mathrm{c} \rightarrow 0$, which shows that the weight averaged hydrodynamic radius, $\left\langle R_{\mathrm{H}}\right\rangle_{\mathrm{w}}$, of the free chains is $26 \mathrm{~nm}$. The size of the $\left\langle R_{\mathrm{H}}\right\rangle_{\mathrm{w}}$ fits well with the form factor modelling, $P(q)_{\mathrm{MCC}}$, seen in Fig. 1a, as it would correspond to a $q$ value of roughly $0.01 \AA^{-1}$ which, in turn, is roughly where the model starts to reach the plateau. The z-averaged hydrodynamic radius, $\left\langle R_{\mathrm{H}}\right\rangle_{z} \approx 100 \mathrm{~nm}$, obtained from DLS is approximately four times larger than $\left\langle R_{\mathrm{H}}\right\rangle_{\mathrm{w}}$, consistent with a broad size distribution of the MCC material. 

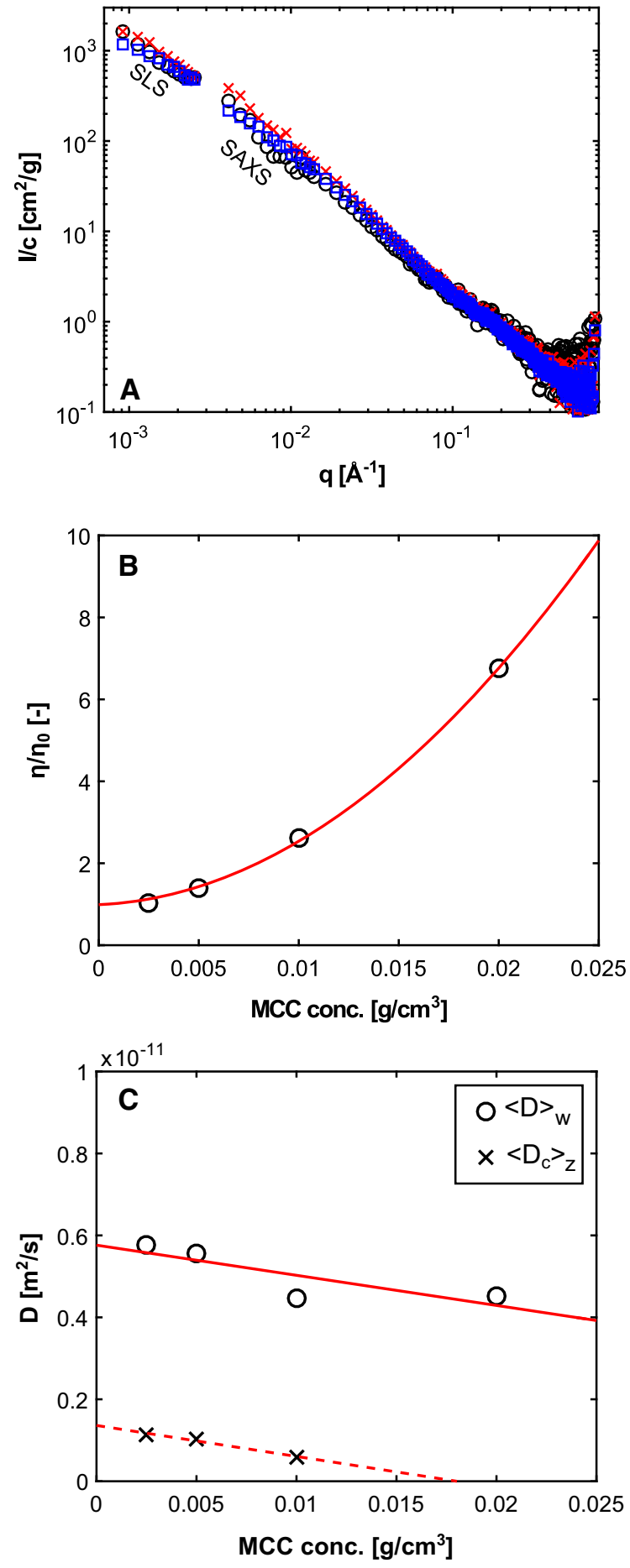

Dissolution studied with ${ }^{1} \mathrm{H}$ NMR

In order to verify that the cold alkali is capable of fully dissolving the Avicel PH101 MCC, samples with
4Fig. 3 a Normalized scattering patterns, $I(q) / c$, for three different cellulose concentrations, $0.0025 \mathrm{~g} / \mathrm{cm}^{3}$ (open circle), $0.0050 \mathrm{~g} / \mathrm{cm}^{3}$ (red cross), and $0.010 \mathrm{~g} / \mathrm{cm}^{3}$ (blue square). b Relative viscosity, $\eta / \eta_{0}$, versus the cellulose concentration. c Average self-diffusion coefficient obtained from NMR (open circle) and collective diffusion coefficient obtained from DLS (cross), plotted as a function of the cellulose concentration

varying concentration of added MCC was prepared and analysed with ${ }^{1} \mathrm{H}-\mathrm{NMR}$. Each sample was prepared individually in order to verify that the dissolution process could be repeated with high accuracy, and in order to check for concentration effects four samples were analysed: a blank with a known concentration of TMABr added to the $2.0 \mathrm{M}$ $\mathrm{NaOD}$ solution and a concentration series of 0.0050 , $0.010,0.020$, and $0.040 \mathrm{~g} / \mathrm{cm}^{3}$. Figure $4 \mathrm{a}$ show the ${ }^{1} \mathrm{H}-\mathrm{NMR}$ spectra for a $0.010 \mathrm{~g} / \mathrm{cm}^{3}$ sample with the $\mathrm{OH}$ peak at $4.5 \mathrm{ppm}$. The ${ }^{1} \mathrm{H}-\mathrm{NMR}$ essentially only detects dissolved MCC as any undissolved material would not contribute to the sharp peaks but would instead only be seen as a slight increase of the baseline. The $-\mathrm{OH}$ peak was used as an internal reference since $\mathrm{NaOD} / \mathrm{D}_{2} \mathrm{O}$ solution gives a fixed amount of protons and any increase of the area of the $-\mathrm{OH}$ peaks comes solely from the increase of cellulose concentration. The concentration of protons in the $-\mathrm{OH}$ peak of the blank could be determined from the known concentration of the added TMABr and each sample analysed was scaled so that their respectively $-\mathrm{OH}$ peak would correspond to the $-\mathrm{OH}$ peak of the blank. Figure $4 \mathrm{~b}$ shows how the area of the $-\mathrm{OH}$ peak changes with the concentration of the added cellulose and this result is then converted into a measured amount of dissolved cellulose in Fig. 4c. The conversion from ${ }^{1} \mathrm{H}$ peak area to concentration is done as

$C_{\mathrm{MCC}}=\frac{I_{\mathrm{MCC}}}{I_{\mathrm{ref}}} \times C_{\mathrm{ref}} \times 12$,

where $I_{\mathrm{MCC}}$ is the sum of the area of all peaks belonging to the MCC spectra divided by seven protons, $I_{\text {ref }}$ is the area of the TMABr peak, $C_{\text {ref }}$ is the concentration of the added TMABr $(\mathrm{mol} / \mathrm{L})$ and 12 is the amount of protons in the TMABr. The data in Fig. $4 \mathrm{c}$ is seen to almost perfectly fit along a straight line up to $0.020 \mathrm{~g} / \mathrm{cm}^{3}$. For the $0.040 \mathrm{~g} / \mathrm{cm}^{3}$ sample, on the other hand, the dissolved amount is only $0.036 \mathrm{~g} / \mathrm{cm}^{3}$, which we identify as a first estimate of the MCC solubility in cold 2.0 M NaOH(aq) (Fig. 4). 

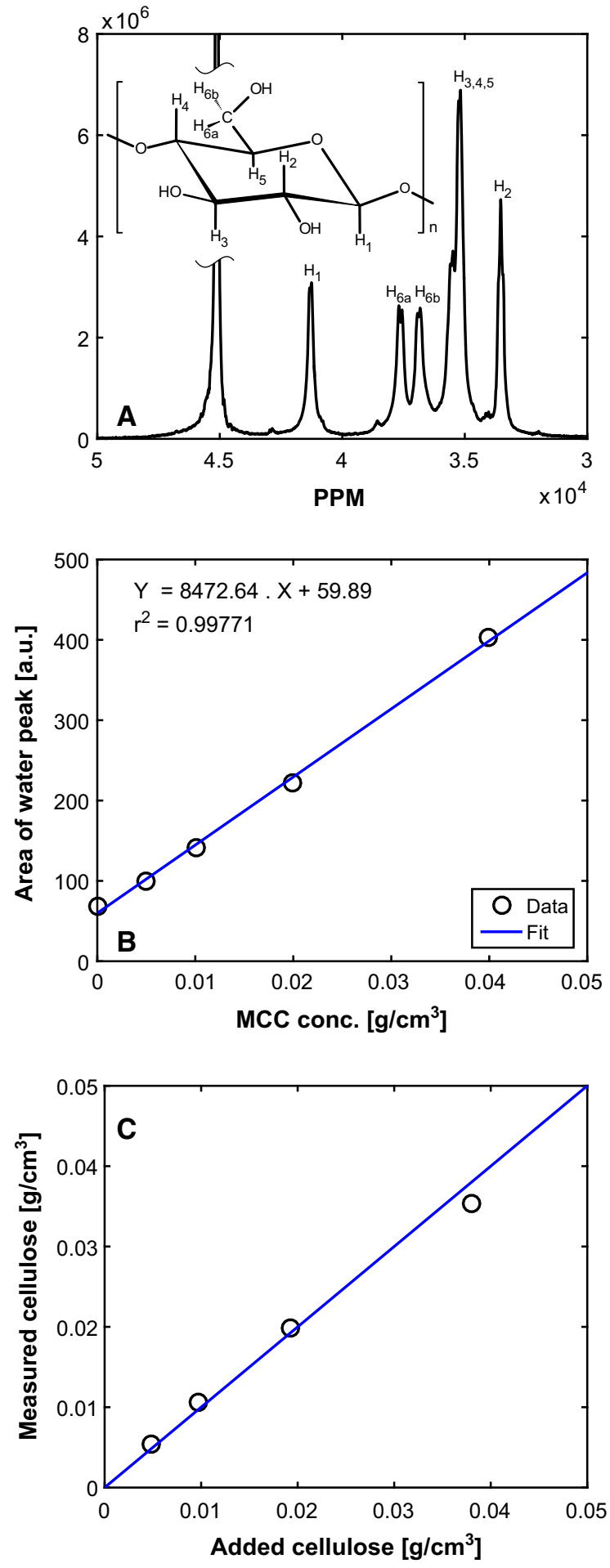

4Fig. 4 a A ${ }^{1} \mathrm{H}-\mathrm{NMR}$ spectrum of a $0.010 \mathrm{~g} / \mathrm{cm}^{3}$ sample dissolved in $2.0 \mathrm{M} \mathrm{NaOH}$. b The integral of the $-\mathrm{OH}$ peak as function of the cellulose concentration, this relation is used as internal reference in order to measure the amount of dissolved MCC. c The concentration of dissolved cellulose versus the cellulose concentration, calibrated using a blank with a known concentration of TMABr

Solvent effects

\section{Effect of $\mathrm{NaOH}$ concentration}

The window for optimal dissolution of cellulose in $\mathrm{NaOH}$ is rather narrow, ranging between 1.5 and 2.5 M (Cai and Zhang 2005; Kihlman et al. 2013) and outside of it, only minute quantities of cellulose is dissolved. To perform the study of the effect of $\mathrm{NaOH}$ concentration, the samples were prepared as $0.020 \mathrm{~g} /$ $\mathrm{cm}^{3}$ at $2.0 \mathrm{M}$ and then diluted to $0.010 \mathrm{~g} / \mathrm{cm}^{3}$ and desired $\mathrm{NaOH}$ concentration by mixing $50 \mathrm{wt} \%$ of the stem solution with $50 \mathrm{wt} \%$ of either water or a solution of $1.0,3.0$, or $4.0 \mathrm{M} \mathrm{NaOH}$. Figure 5a shows the results from the SAXS measurement of the samples, where the data have been corrected for the difference in scattering length density $(\Delta \varrho)$ between the samples. The fractal aggregation model (Teixeira 1988) was fitted to the data by varying the fractal dimension, $D$, and the correlation length, $\xi$, using a fixed radius of $0.25 \mathrm{~nm}$. The scattering intensity from the SAXS measurements increases when the $\mathrm{NaOH}$ concentration deviates from $2.0 \mathrm{M}$, as shown in Fig. 5a. The intensity for 1.5 and $2.5 \mathrm{M}$ differs only slightly from the $2.0 \mathrm{M}$ case; however, to reproduce such small difference in the data modelling it is required to significantly alter the fractal dimension, $D$ (Fig. 5b). In the case of 1.0 and $3.0 \mathrm{M} \mathrm{NaOH}$ solutions, the SAXS intensity increases further and in order to model the data, the fractal dimension needs to be altered even more. It should be noted that there are also some minor alterations in correlation length, but in general it stays fairly close to the same for all solvent $\mathrm{NaOH}(\mathrm{aq})$ concentrations except $1.0 \mathrm{M}$, which is significantly larger. Comparing the scattering to the rheology data shows highly similar results. In the cases of $1.5-2.5 \mathrm{M}$ solutions, all of the samples are Newtonian and exhibit low variation while the 1.0 and the $3.0 \mathrm{M}$ solutions 

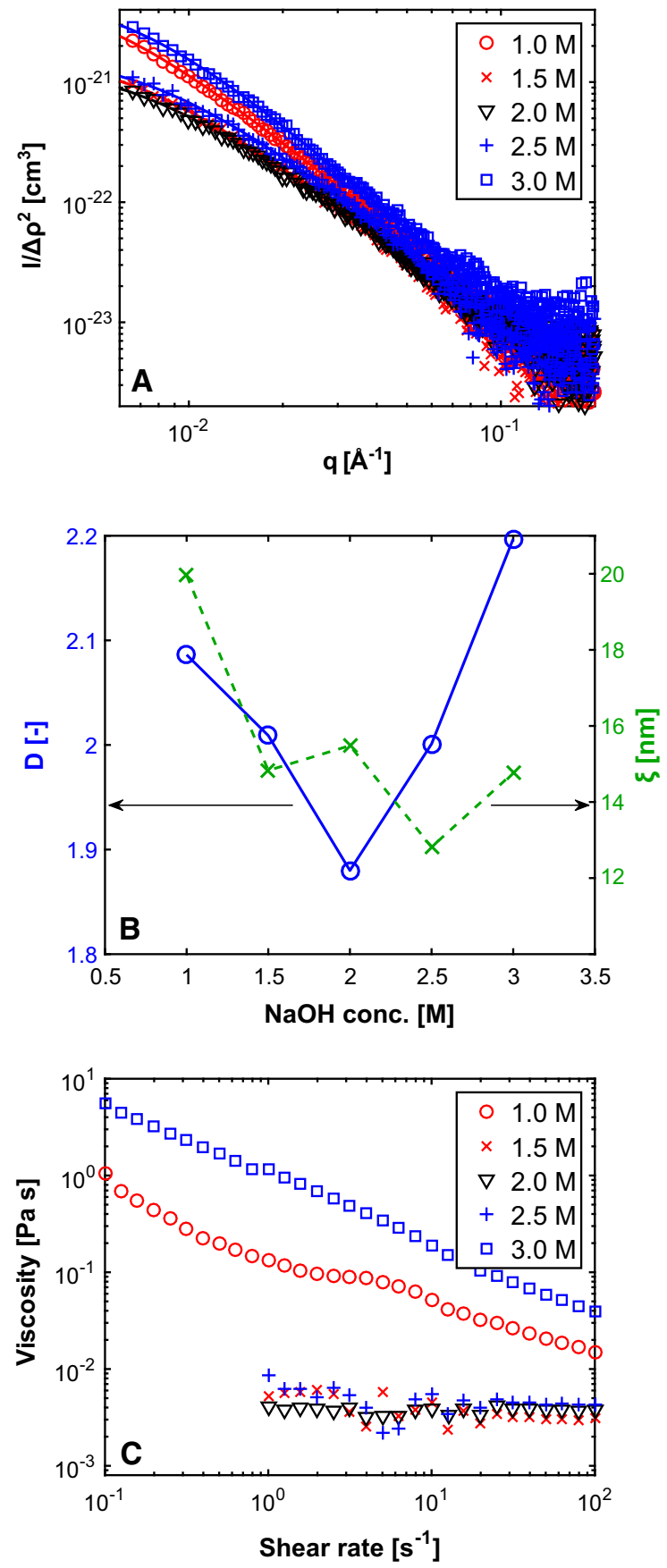

Fig. 5 SAXS and viscosity results from a $0.010 \mathrm{~g} / \mathrm{cm}^{3}$ cellulose solution in different concentrations of $\mathrm{NaOH}(\mathrm{aq})$, varying from 1.0 to $3.0 \mathrm{M}$. a SAXS patterns. The solid lines represent fractal model values. $\mathbf{b}$ Fits of the fractal dimension, $D$, and the correlation length, $\xi$, obtained (from Eq. 6) at different concentrations of $\mathrm{NaOH}(\mathrm{aq})$. c Viscosity as a function of the shear rate of the samples become shear thinning. The shear thinning behaviour is caused by a gelation of the system as the MCC starts precipitate and to form stronger aggregates. The exact mechanism for the gelation is still uncertain and the gelation is somewhat peculiar since the MCC concentration is below the critical overlap concentration, $C^{*}$ and, therefore, should not be able to percolate the entire volume.

\section{Effect of urea}

Urea has been discussed as a positive co-solvent for cold alkali (Cai and Zhang 2005; Jin et al. 2007; Wernersson et al. 2015; Zhang et al. 2010), and the addition is reported to promote the dissolution process. In Fig. 6, the effect of urea upon MCC is demonstrated for the system used in this paper. Urea was added to the solvent prior to MCC dissolution in such a way as to keep the $\mathrm{NaOH}$ at a constant $2.0 \mathrm{M}$ concentration. The difference between three different urea concentrations was investigated: $0,1.8$, and $3.6 \mathrm{M}$, and the result seemed to be inconclusive. No significant difference can be seen between 0 and $1.8 \mathrm{M}$ urea for any of the MCC concentrations. Looking at the $3.6 \mathrm{M}$ urea addition, it appears as it there is a slight increase in scattering intensity for $0.0025 \mathrm{~g} / \mathrm{cm}^{3}$ MCC (Fig. 6a), but the curve is offset only with a constant and not by any significant amount which suggests that the difference is caused by the accuracy of the measurement of the water scattering for the absolute scale of the capillary used. When it comes to the $0.0050 \mathrm{~g} /$ $\mathrm{cm}^{3}$ (Fig. 6b) and $0.010 \mathrm{~g} / \mathrm{cm}^{3}$ (Fig. 6c), the results are contradictory. For the $0.0050 \mathrm{~g} / \mathrm{cm}^{3}$ sample, it appears as if the addition of urea results in a lowered scattering intensity whereas for the $0.010 \mathrm{~g} / \mathrm{cm}^{3}$ case, there is an increase. Such inconclusive results indicate that the addition of urea does not significantly influence the structure of the dissolved MCC.

Temperature dependence

The temperature treatment of MCC in $2.0 \mathrm{M} \mathrm{NaOH}$ was performed so that it started at $25^{\circ} \mathrm{C}$ and then was increased up to $45^{\circ} \mathrm{C}$ in $5^{\circ} \mathrm{C}$ steps, and between each step, the temperature was lowered down to $25^{\circ} \mathrm{C}$ again to see whether any changes were reversible. The effect of the temperature treatment becomes 

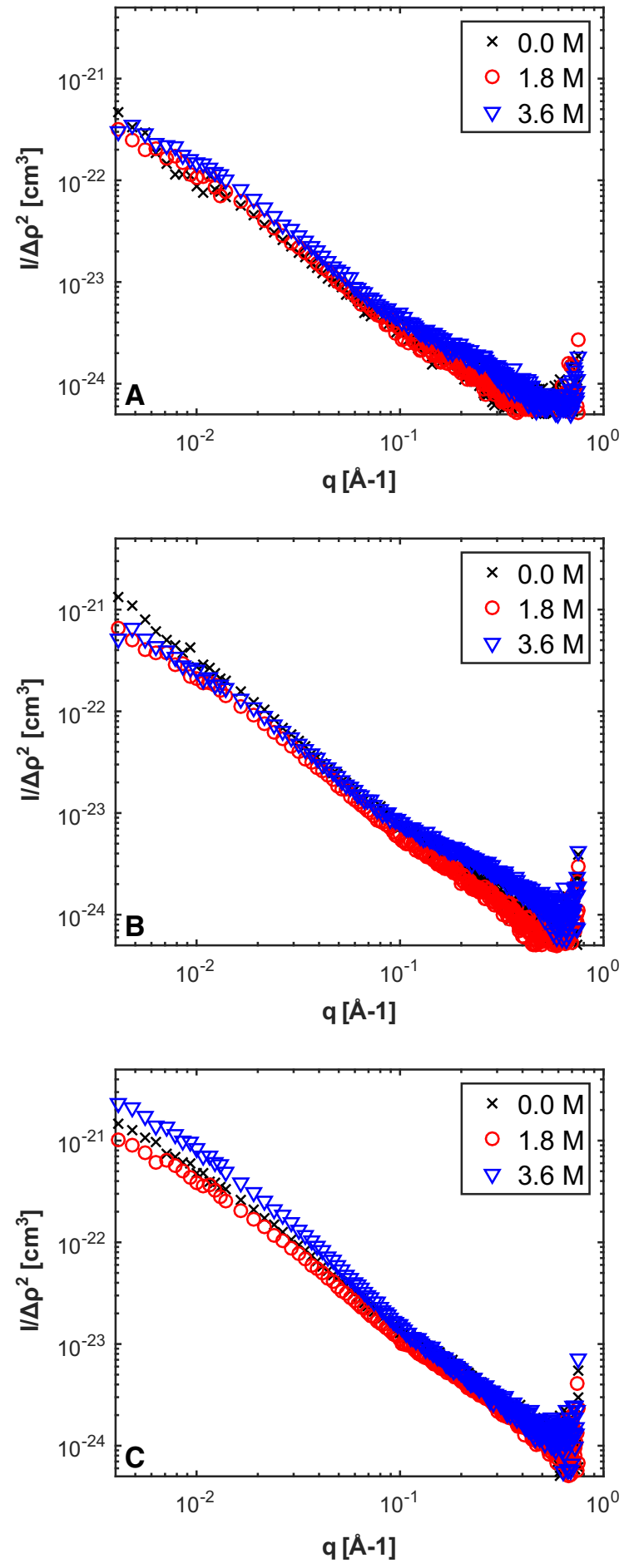

Fig. 6 SAXS patterns obtained for different cellulose concentration in $2.0 \mathrm{M} \mathrm{NaOH}(\mathrm{aq})$ containing with different concentrations of urea: $0 \mathrm{M}$ (cross), $1.8 \mathrm{M}$ (open circle) and as $3.6 \mathrm{M}$ (blue upside down triangle). The cellulose concentrations are a $0.0025 \mathrm{~g} / \mathrm{cm}^{3}, \mathbf{b} 0.0050 \mathrm{~g} / \mathrm{cm}^{3}$, and $\mathbf{c} 0.010 \mathrm{~g} / \mathrm{cm}^{3} \mathrm{MCC}$ noticeable in the SAXS data at around $35^{\circ} \mathrm{C}$ as an increase in intensity at low $q$, whereas the rheology data do not indicate any significant changes until the sample was heated to $40^{\circ} \mathrm{C}$, which can be seen in Fig. 7a, b. The solid lines in Fig. 7a represent the heating step, while the dashed lines represent the subsequent cooling to $25^{\circ} \mathrm{C}$, and as the solid and dashed lines more or less overlap perfectly for each temperature, this is an indication that the aggregations induced by the heating are irreversible.

As opposed to the effect of the $\mathrm{NaOH}$ concentration, where the curves in Fig. 5a increase at a relatively high $\mathrm{q}$, the effect of the temperature causes the curves in Fig. 7 to bend at roughly $3.5 \times 10^{-2} \AA^{-1}$. Since the curves deviate at lower $\mathrm{q}$, but not at higher $\mathrm{q}$, that
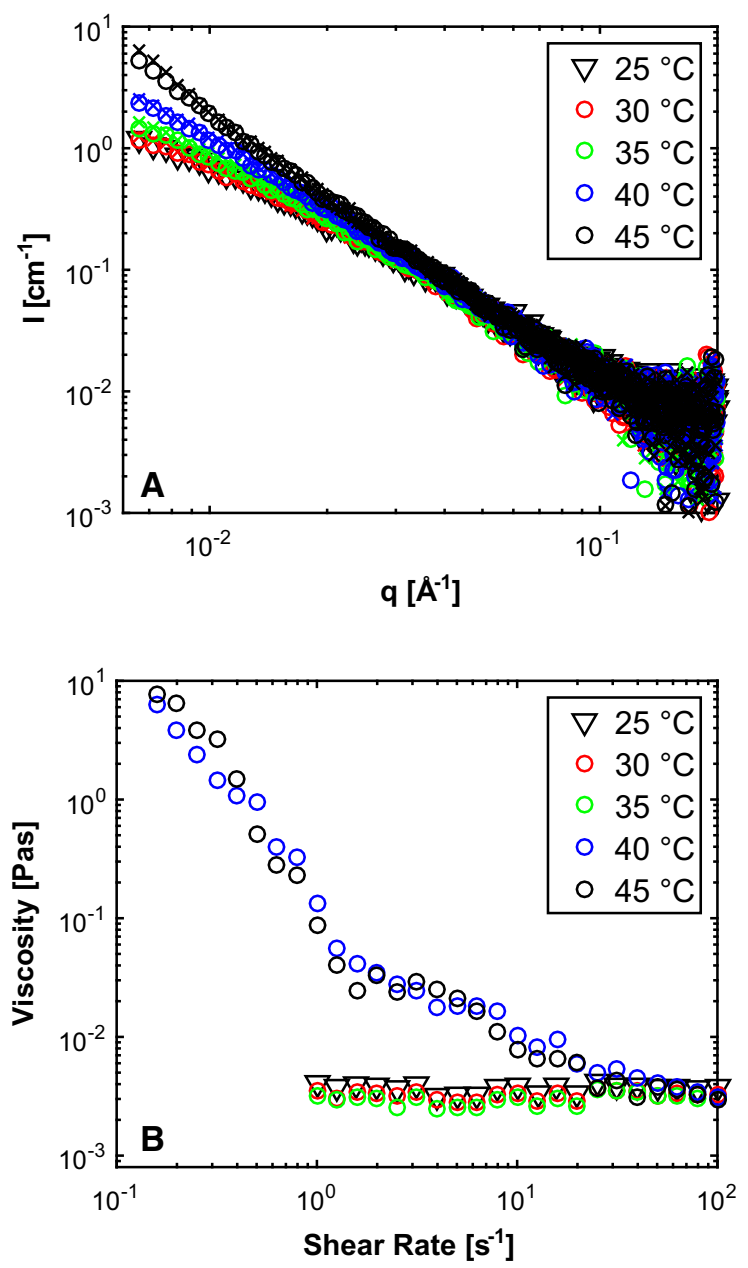

Fig. 7 The temperature dependence on a $0.010 \mathrm{~g} / \mathrm{cm}^{3}$ sample in $2.0 \mathrm{M}$ as measured by $\mathbf{a}$ SAXS and $\mathbf{b}$ rheology. Each increase in temperature is represented by open circled while times indicates the subsequent decrease back to $25^{\circ} \mathrm{C}$ 
means that the cellulose chains clump together and create a cluster, but that the $R_{g}$ of the individual chains does not change significantly. The bend in the data indicates the mesh size $(\xi)$ of the cluster, and a value of $3.5 \times 10^{-2} \AA^{-1}$ corresponds to $180 \AA$, showing that $\xi$ is on the same length scale as roughly half of $\left\langle R_{g}\right\rangle$ of the individual cellulose chains.

The rheology measurements at 40 and $45^{\circ} \mathrm{C}$ indicate that the sample forms an irreversible gel. The exact reason for the gelation is currently unknown, but the $C^{*}$ appears to be dependent on temperature (Roy et al. 2003), and it is not impossible that $C^{*}$ is lowered enough for the solution to percolate the system at $1 \mathrm{~g} /$ $\mathrm{cm}^{3}$. It has also been shown that the temperature induced gelation of cellulose in alkaline solvent is very time dependent, where the gelation time varies from minutes to days depending on temperature (Cai and Zhang 2006).

The temperature effect has also been studied with static light scattering and the most noticeable effect is that the intensity increases and becomes more and more noisy with increasing temperature. Figure 8a illustrates this by showing the difference between 25 and $45^{\circ} \mathrm{C}$. The data from the higher temperature is a bit too noisy to accurately determine the slope, but it appears as if the data remain parallel within this $q$ range, as opposed to the lower part of the SAXS $q$ range, where the slope increases with temperature. One way to analyse the noisy data is to compare how the variance changes with temperature. In Fig. 8b, such analysis is presented as $\sigma /\langle I\rangle$ as a function of temperature. It can be seen that the fluctuations increases slowly up until $40^{\circ} \mathrm{C}$, after which the data become highly noisy. This suggests that strong attractive forces are acting upon the cellulose, something which does not agree with the result of Kamide et al. (1987). These strong attractive forces cause the cellulose to aggregate into fewer, larger clusters with increasing temperature, which is the reason for the larger variance in the data at higher temperatures. In neither Figs. 7 or 8 is the effect decreased when the samples are cooled down to $25^{\circ} \mathrm{C}$ after each step, which indicates irreversible aggregation. An explanation to how the chains aggregate would, therefore, be that the chains bind together at the edges and create crystalline areas, which causes the aggregation to be irreversible, and the network they form is spaced out by the $R_{g}$ of the individual chains.
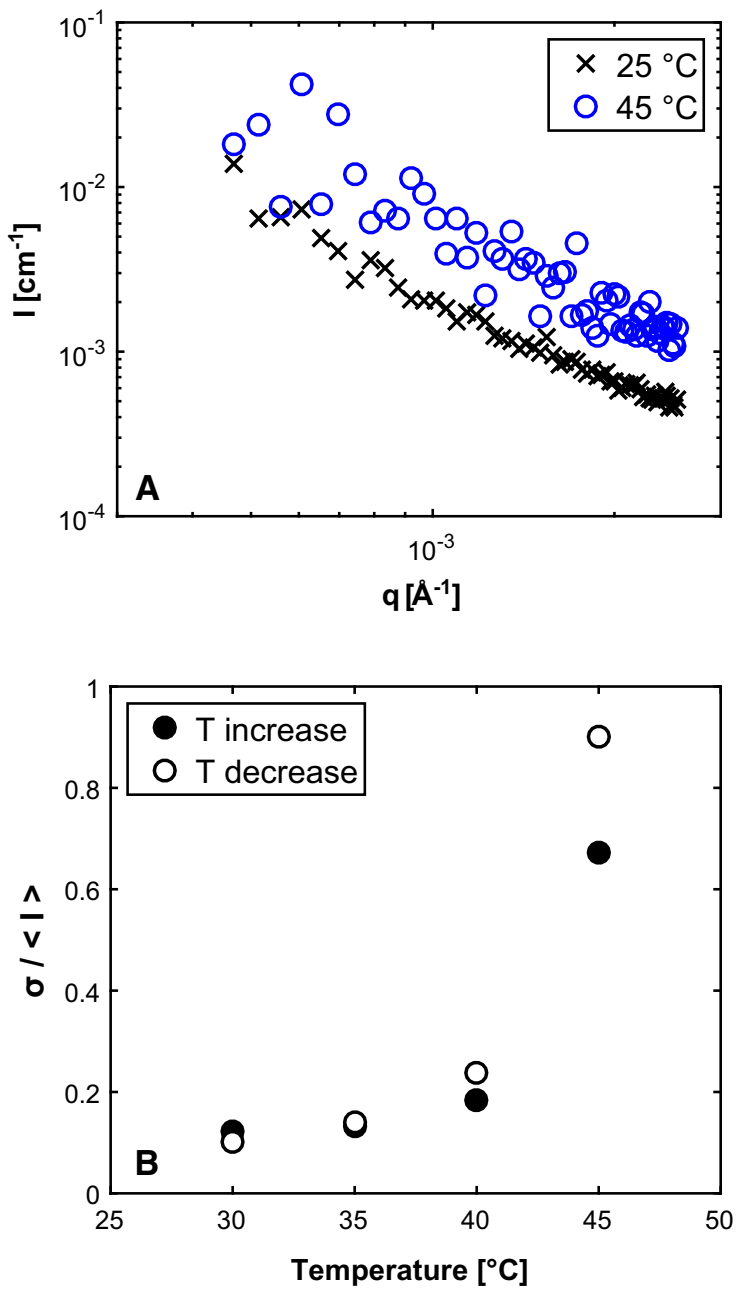

Fig. 8 a Light scattering patterns from a $0.010 \mathrm{~g} / \mathrm{cm}^{3}$ sample in $2.0 \mathrm{M} \mathrm{NaOH}$ (aq), at 25 and $45^{\circ} \mathrm{C}$, respectively. b Normalized variance of the data as a function of temperature

\section{Conclusions}

We have shown here that MCC can be fully molecularly dissolved in $\mathrm{NaOH}$ and that the samples are stable within the $q$ range of $6 \times 10^{-3}-2 \times 10^{-1} \AA^{-1}$ for up to 2 months at room temperature $\left(22 \pm 1{ }^{\circ} \mathrm{C}\right)$ at these low concentrations. The dissolution was verified with NMR, and it was also seen that it is possible to use the $-\mathrm{OH}$ peak as an internal standard when measuring the amount of dissolved MCC in $\mathrm{NaOH}$. The MCC dissolved in $2.0 \mathrm{M} \mathrm{NaOH}$ exhibit dissolved and essentially free cellulose chains in solution, except for a very few, but large aggregates. These clusters of cellulose strands are strong enough not to disappear 
upon dilution, which can be explained because the strands in the aggregates are bound together in crystalline patches. While the clusters bend light and gives and apparent size of $>1 \mu \mathrm{m}$ in scattering, NMR and rheology clearly show that the chains are free to move around and that no species are larger than a 400. The solutions gel upon alteration of the $\mathrm{NaOH}$ concentration or a change in temperature, where the two different treatments give rise to two different types of structures. Addition of urea has been shown to give inconclusive results, as no significant effect was observed upon the dissolution of cellulose. These results give greater understanding of the behaviour of cellulose in cold alkali solutions and yields and insight in how potential fibre spinning of cellulose from $\mathrm{NaOH}$ can be achieved.

Acknowledgments This work was supported by Bo Rydin Foundation and by Södra Skogsgarnas Stiftelse through the research program Avancell. We thank Caroline Löfgren and Majid Ghasemi for the analysis of the cellulose size distribution. This work benefited from SasView software, originally developed by the DANSE project under NSF award DMR-0520547

Open Access This article is distributed under the terms of the Creative Commons Attribution 4.0 International License (http:// creativecommons.org/licenses/by/4.0/), which permits unrestricted use, distribution, and reproduction in any medium, provided you give appropriate credit to the original author(s) and the source, provide a link to the Creative Commons license, and indicate if changes were made.

\section{References}

Alves L, Medronho B, Antunes FE, Topgaard D, Lindman B (2016) Dissolution state of cellulose in aqueous systems. 1. Alkaline solvents. Cellulose 23(1):247-258

Behrens MA, Holdaway JA, Nosrati P, Olsson U (2016) On the dissolution state of cellulose in aqueous tetrabutylammonium hydroxide solutions. RSC Adv 6(36):30199-30204

Budtova T, Navard P (2016) Cellulose in $\mathrm{NaOH}$-water based solvents: a review. Cellulose 23(1):5-55

Cai J, Zhang L (2005) Rapid dissolution of cellulose in LiOH/ urea and $\mathrm{NaOH} /$ urea aqueous solutions. Macromol Biosci 5(6):539-548

Cai J, Zhang L (2006) Unique gelation behavior of cellulose in $\mathrm{NaOH} /$ urea aqueous solution. Biomacromolecules 7(1): 183-189

Chen W-R, Butler PD, Magid LJ (2006) Incorporating intermicellar interactions in the fitting of SANS data from cationic wormlike micelles. Langmuir 22:6539-6548
Chen X, Burger C, Wan F, Zhang J, Rong L, Hsiao BS, Chu B, Cai J, Zhang L (2007) Structure study of cellulose fibers wet-spun from environmentally friendly $\mathrm{NaOH} / \mathrm{urea}$ aqueous solutions. Biomacromolecules 8(6):1918-1926

Gentile L, Olsson U (2016) Cellulose-solvent interactions from self-diffusion NMR. Cellulose 23(4):2753-2758

Gubitosi M, Duarte H, Gentile L, Olsson U, Medronho B (2016) On cellulose dissolution and aggregation in aqueous tetrabutylammonium hydroxide. Biomacromolecules 17(9):2873-2881

Huggins ML (1942) The viscosity of dilute solutions of longchain molecules. IV. Dependence on concentration. J Am Chem Soc 64(11):2716-2718

Jin H, Chunxi Z, Gu L (2007) Direct dissolution of cellulose in $\mathrm{NaOH} /$ thiourea/urea aqueous solution. Carbohydr Res 342(6):851-858

Kamide K, Saito M, Kowsaka K (1987) Temperature dependence of limiting viscosity number and radius of gyration for cellulose dissolved in aqueous $8 \%$ sodium hydroxide solution. Polym J 19(10):1173-1181

Kihlman M, Medronho BF, Romano AL, Germgård U, Lindman B (2013) Cellulose dissolution in an alkali based solvent: influence of additives and pretreatments. J Braz Chem Soc 24(2):295-303

Liebert T (2010) Kapitel 01-cellulose solvents—remarkable history, bright future. Cellulose solvents: for analysis, shaping and chemical modification. ACS Symp Ser Am Chem Soc 1033:3-54. doi:10.1021/bk-2010-1033.ch001

Medronho B, Lindman B (2014a) Competing forces during cellulose dissolution: from solvents to mechanisms. Curr Opin Colloid Interface Sci 19(1):32-40

Medronho B, Lindman B (2014b) Brief overview on cellulose dissolution/regeneration interactions and mechanisms. Adv Colloid Interface Sci 222:502-508

Pedersen JS, Schurtenberger P (1996) Scattering functions of semiflexible polymers with and without excluded volume effects. Macromolecules 29:7602-7612

Price WS (2009) NMR studies of translational motion: principles and applications. Cambridge University Press, Cambridge

Roy C, Budtova T, Navard P (2003) Rheological properties and gelation of aqueous cellulose- $\mathrm{NaOH}$ solutions. Biomacromolecules 4:259-264

Schulz L, Seger B, Burchard W (2000) Structures of cellulose in solution. Macromol Chem Phys 201(15):2008-2022

Teixeira J (1988) Small-angle scattering by fractal systems. J Appl Crystallogr 21:781-785

Van Loon LR, Glaus MA (1997) Review of the kinetics of alkali degradation of cellulose. J Environ Polym Degrad 5(2):97-109

Wernersson E, Stenqvist B, Lund M (2015) The mechanism of cellulose solubilization by urea studied by molecular simulation. Cellulose 22(2):991-1001

Zhang S, Li F-X, Yu J, Hsieh Y-L (2010) Dissolution behaviour and solubility of cellulose in $\mathrm{NaOH}$ complex solution. Carbohydr Polym 81(3):668-674 